\title{
THE ZEROS DISTRIBUTION OF SOLUTIONS OF HIGHER ORDER DIFFERENTIAL EQUATIONS IN AN ANGULAR DOMAIN
}

\author{
Zhibo Huang and Zongxuan Chen
}

\begin{abstract}
In this paper, we investigate the zeros distribution and Borel direction for the solutions of linear homogeneous differential equation

$$
f^{(n)}+A_{n-2}(z) f^{(n-2)}+\cdots+A_{1}(z) f^{\prime}+A_{0}(z) f=0(n \geq 2)
$$

in an angular domain. Especially, we establish a relation between a cluster ray of zeros and Borel direction.
\end{abstract}

\section{Introduction}

We use the standard notations from Nevanlinna theory in this paper (see $[4,5,13])$.

The study of the zeros distribution of solutions of a linear differential equation is one of the difficult aspects in the complex oscillation theory of differential equations. However, different authors have obtained some results (see $[3,7,10,11,12,14])$.

In order to state our results, we give some definitions.

Let $g(z)$ be an entire function in the plane and let $\arg z=\theta \in[0,2 \pi)$ be a ray. We denote an angular domain and a sectorial domain, for any $\alpha<\beta$, respectively,

$$
\begin{aligned}
& \Omega(\alpha, \beta)=\{z|\alpha \leq \arg z \leq \beta,| z \mid>0\}, \\
& \Omega((\alpha, \beta), r)=\{z|z \in \Omega(\alpha, \beta),| z \mid<r\} .
\end{aligned}
$$

Moreover, we define the sectorial maximus modulus for an entire function by

$$
M(\Omega((\alpha, \beta), r), g)=\sup \{|g(z)|: z \in \Omega((\alpha, \beta), r)\} .
$$

Received February 5, 2008; Revised March 19, 2009.

2000 Mathematics Subject Classification. 30D35.

Key words and phrases. zeros distribution, linear differential equation, hyper order, Borel direction.

This project was supported by the National Natural Science Foundation of China (No.10871076), and also partly supported by the School of Mathematical Sciences Foundation of SCNU, China. 
The order $\sigma(\Omega(\alpha, \beta), g)$ of $g(z)$ in the angular domain $\Omega(\alpha, \beta)$ is defined by

$$
\sigma(\Omega(\alpha, \beta), g)=\varlimsup_{r \rightarrow \infty} \frac{\log \log M(\Omega((\alpha, \beta), r), g)}{\log r} .
$$

The hyper order $\sigma_{2}(\Omega(\alpha, \beta), g)$ of $g(z)$ in the angular domain $\Omega(\alpha, \beta)$ is defined by

$$
\sigma_{2}(\Omega(\alpha, \beta), g)=\varlimsup_{r \rightarrow \infty} \frac{\log \log \log M(\Omega((\alpha, \beta), r), g)}{\log r}
$$

Let $n(\Omega((\alpha, \beta), r), g=a)$ be the number of $a$-points, i.e., roots of the equation $g(z)=a$ in the sectorial domain $\Omega((\alpha, \beta), r)$.

The exponent of convergence of zero sequence of $g(z)-a$ in the angular domain $\Omega(\alpha, \beta)$ is defined by

$$
\lambda(\Omega(\alpha, \beta), g=a)=\varlimsup_{r \rightarrow \infty} \frac{\log n(\Omega((\alpha, \beta), r), g=a)}{\log r} .
$$

The hyper order exponent of convergence of zero sequence of $g(z)-a$ in the angular domain $\Omega(\alpha, \beta)$ is defined by

$$
\lambda_{2}(\Omega(\alpha, \beta), g=a)=\varlimsup_{r \rightarrow \infty} \frac{\log \log n(\Omega((\alpha, \beta), r), g=a)}{\log r} .
$$

We also denote, for each $\varepsilon>0$, the exponent and the hyper order exponent of convergence of zero sequence of $g(z)$ in the angular domain $\Omega(\theta-\varepsilon, \theta+\varepsilon)$ by $\lambda_{\theta, \varepsilon}(g)$ and $\lambda_{2, \theta, \varepsilon}(g)$, respectively, i.e.,

$$
\lambda_{\theta, \varepsilon}(g)=\varlimsup_{r \rightarrow \infty} \frac{\log n(\Omega((\theta-\varepsilon, \theta+\varepsilon), r), g=a)}{\log r},
$$

and

$$
\lambda_{2, \theta, \varepsilon}(g)=\varlimsup_{r \rightarrow \infty} \frac{\log \log n(\Omega((\theta-\varepsilon, \theta+\varepsilon), r), g=a)}{\log r},
$$

and by $\lambda_{\theta}(g)=\lim _{\varepsilon \rightarrow 0} \lambda_{\theta, \varepsilon}(g)$ and $\lambda_{2, \theta}(g)=\lim _{\varepsilon \rightarrow 0} \lambda_{2, \theta, \varepsilon}(g)$, respectively.

Our proofs also require the Nevanlinna characteristic function for an angular domain (see $[3,9,10]$ ). If $0<\beta-\alpha \leq 2 \pi$ and $k=\frac{\pi}{\beta-\alpha}$ and $g(z)$ is meromorphic in the angular domain $\Omega(\alpha, \beta)$, we denote

$$
\begin{aligned}
& A_{\alpha, \beta}(r, g)=\frac{k}{\pi} \int_{1}^{r}\left(\frac{1}{t^{k}}-\frac{t^{k}}{r^{2 k}}\right)\left\{\log ^{+}\left|g\left(t e^{i \alpha}\right)\right|+\log ^{+}\left|g\left(t e^{i \beta}\right)\right|\right\} \frac{d t}{t} \\
& B_{\alpha, \beta}(r, g)=\frac{2 k}{\pi r^{k}} \int_{\alpha}^{\beta} \log ^{+}\left|g\left(r e^{i \theta}\right)\right| \sin k(\theta-\alpha) d \theta \\
& C_{\alpha, \beta}(r, g)=2 \sum_{1<\left|b_{v}\right|<r}\left(\frac{1}{\left|b_{v}\right|^{k}}-\frac{\left|b_{v}\right|^{k}}{r^{2 k}}\right) \sin k\left(\beta_{v}-\alpha\right) ; \\
& D_{\alpha, \beta}(r, g)=A_{\alpha, \beta}(r, g)+B_{\alpha, \beta}(r, g) ; \\
& S_{\alpha, \beta}(r, g)=A_{\alpha, \beta}(r, g)+B_{\alpha, \beta}(r, g)+C_{\alpha, \beta}(r, g)
\end{aligned}
$$


where $b_{v}=\left|b_{v}\right| e^{i \beta_{v}}(v=1,2, \ldots)$ are the poles of $g(z)$ in the angular domain $\Omega(\alpha, \beta)$, counting multiplicities. If we only consider the distinct poles of $g(z)$, we denote the corresponding angular counting function by $\bar{C}_{\alpha, \beta}(r, g)$. $S_{\alpha, \beta}(r, g)$ and $C_{\alpha, \beta}(r, g)$ are called the Nevanlinna's angular characteristic function and the angular counting function, respectively. The sectorial hyper order $\rho_{2}(\Omega(\alpha, \beta), g)$ of $g(z)$ in the angular domain $\Omega(\alpha, \beta)$ will be defined by

$$
\rho_{2}(\Omega(\alpha, \beta), g)=\varlimsup_{r \rightarrow \infty} \frac{\log \log S_{\alpha, \beta}(r, g)}{\log r} .
$$

In [7], we considered the equation

$$
f^{\prime \prime}+A(z) f=0
$$

where $A(z)$ is an entire function with order $\sigma(A)=+\infty$ and the hyper order $\sigma_{2}(A)=0$. We have obtained the following results.

Theorem 1.1. Let $A(z)$ be an entire function with order $\sigma(A)=+\infty$ and the hyper order $\sigma_{2}(A)=0$ and let $f_{1}$ and $f_{2}$ be two linearly independent solutions of (1.1). Set $E=f_{1} f_{2}$. Then $\lambda_{2, \theta}(E)=+\infty$ if and only if

$$
\varlimsup_{r \rightarrow \infty} \frac{\log \log \log M(\Omega((\theta-\varepsilon, \theta+\varepsilon), r), E)}{\log r}=\varlimsup_{r \rightarrow \infty} \frac{\log \log S_{\theta-\varepsilon, \theta+\varepsilon}(r, E)}{\log r}=+\infty
$$

for all $\varepsilon>0$.

Theorem 1.2. Let $A(z)$ be an entire function with order $\sigma(A)=+\infty$ and the hyper order $\sigma_{2}(A)=0$ and let $f_{1}$ and $f_{2}$ be two linearly independent solutions of (1.1). Set $E=f_{1} f_{2}$. Suppose that the hyper order exponent of convergence of zero sequence of $E$ is $+\infty$. Then a ray $\arg z=\theta$ from the origin is a Borel direction of $E$ with the hyper order $+\infty$ and $\rho_{2}(\Omega(\theta-\varepsilon, \theta+\varepsilon), E)=+\infty$ if and only if $\lambda_{2, \theta}(E)=+\infty$.

\section{The zeros distribution in an angular domain}

In order to relate our result, we need the followings.

Suppose that $g(z)$ is analytic. Then $g(z)$ has the power series representation

$$
g(z)=\sum_{n=0}^{\infty} a_{n} z^{n} \quad(0 \leq|z|<\infty) .
$$

Denote maximum item and center index of $g(z)$ by $\mu(r)$ and $\nu(r)$, respectively, i.e.,

$$
\mu(r)=\max _{n \geq 0}\left\{\left|a_{n}\right| r^{n}\right\}
$$

and

Setting $a=\max _{n \geq 0}\left\{\left|a_{n}\right|\right\}$, we have

$$
\nu(r)=\max \left\{m: \mu(r)=\left|a_{m}\right| r^{m}\right\} .
$$

$$
\left|a_{n}\right| r^{n} \leq \mu(r) \leq a r^{\nu(r)} .
$$


Lemma 2.1 ([6, p. 18]). Suppose that $g(z)$ is analytic. Then for $r<R$ and $\mu(r)>1$,

$$
M(r, g) \leq \mu(r)\{1+\log M(R, g)\} \frac{2 R}{R-r} .
$$

On the other hand, under the hypotheses of Lemma 2.1, we have, for all $0 \leq r<R$,

$$
T(r, g) \leq \log M(r, g) \leq \frac{R+r}{R-r} T(R, g) .
$$

Together with Lemma 2.1 in which we set $R=2 r$, we obtain

$$
\begin{aligned}
T(r, g) & \leq \log \mu(r)+\log \log M(2 r, g)+O(1) \\
& \leq \nu(r) \log r+\log T(4 r, g)+O(1) .
\end{aligned}
$$

Lemma $2.2([8])$. Let $f_{1}, f_{2}, \ldots, f_{n}$ be $n$ linearly independent meromorphic solutions of the equation

$$
f^{(n)}+A_{n-1}(z) f^{(n-1)}+\cdots+A_{1}(z) f^{\prime}+A_{0}(z) f=0, n \geq 2,
$$

with meromorphic coefficients. Then the Wronskian determinant

$$
W=W\left(f_{1}, f_{2}, \ldots, f_{n}\right)=\left|\begin{array}{rrrr}
f_{1} & f_{2} & \cdots & f_{n} \\
f_{1}^{\prime} & f_{2}^{\prime} & \cdots & f_{n}^{\prime} \\
\vdots & \vdots & \ddots & \vdots \\
f_{1}^{(n-1)} & f_{2}^{(n-1)} & \cdots & f_{n}^{(n-1)}
\end{array}\right|,
$$

satisfying the differential equation $W^{\prime}+A_{n-1}(z) W=0$. Specially, if $A_{n-1}(z)$ is an entire function, then for some $c \in \mathbb{C}, W\left(f_{1}, f_{2}, \ldots, f_{n}\right)=c \exp (-\varphi)$, where $\varphi$ is a primitive function of $A_{n-1}(z)$.

Lemma $2.3([3])$. Suppose that $g(z)(\not \equiv$ constant $)$ is meromorphic in the plane and that $\Omega(\alpha, \beta)$ is an angular domain, where $0<\beta-\alpha \leq 2 \pi$. Then

(i) for any complex number $a \neq \infty$,

$$
S_{\alpha, \beta}\left(r, \frac{1}{g-a}\right)=S_{\alpha, \beta}(r, g)+O(1)
$$

(ii) for any $r<R$,

$$
A_{\alpha, \beta}\left(r, \frac{g^{\prime}}{g}\right) \leq K\left\{\left(\frac{R}{r}\right)^{k} \int_{1}^{R} \frac{\log T(t, g)}{t^{1+k}} d t+\log \frac{r}{R-r}+\log \frac{R}{r}+1\right\},
$$

and

$$
B_{\alpha, \beta}\left(r, \frac{g^{\prime}}{g}\right) \leq \frac{4 k}{r^{k}} m\left(r, \frac{g^{\prime}}{g}\right),
$$

where $k=\frac{\pi}{\beta-\alpha}$ and $K$ is a positive constant not depending on $r$ and $R$. 
Lemma $2.4([7])$. Suppose that $\Omega(\alpha, \beta)$ and $\Omega\left(\alpha^{\prime}, \beta^{\prime}\right)$ are two angular domains such that $\alpha<\alpha^{\prime}<\beta^{\prime}<\beta$ and that $g(z)$ is an entire function with

$$
\varlimsup_{r \rightarrow \infty} \frac{\log \log \log M(r, g)}{\log r}=\varlimsup_{r \rightarrow \infty} \frac{\log \log S_{\alpha^{\prime}, \beta^{\prime}}(r, g)}{\log r}=\sigma_{2}\left(\Omega\left(\alpha^{\prime}, \beta^{\prime}\right), g\right),
$$

and

$$
\varlimsup_{r \rightarrow \infty} \frac{\log \log \log \log M(r, g)}{\log r}=0 .
$$

Then we have for every finite complex a with at most one exception

$$
\varlimsup_{r \rightarrow \infty} \frac{\log \log n(\Omega((\alpha, \beta), r), g=a)}{\log r}=\sigma_{2}\left(\Omega\left(\alpha^{\prime}, \beta^{\prime}\right), g\right) .
$$

Now we consider the equation

$$
f^{(n)}+A_{n-2}(z) f^{(n-2)}+\cdots+A_{1}(z) f^{\prime}+A_{0}(z) f=0, n \geq 2,
$$

where $A_{j}(z)(j=0,1, \ldots, n-2)$ are entire functions. Whether there has result similar to Theorem 1.1. About this, we have the following result.

Theorem 2.5. Let $A_{j}(z)(j=0,1, \ldots, n-2)$ be entire functions with order $\sigma\left(A_{j}\right)=+\infty$ and the hyper order $\sigma_{2}\left(A_{j}\right)=0(j=0,1,2, \ldots, n-2)$, and let $f_{1}, f_{2}, \ldots, f_{n}$ be $n$ linearly independent solutions of (2.2). Set $E=f_{1} f_{2} \cdots f_{n}$. Then $\lambda_{2, \theta}(E)=+\infty$ if and only if

$\varlimsup_{r \rightarrow \infty} \frac{\log \log \log M(\Omega((\theta-\varepsilon, \theta+\varepsilon), r), E)}{\log r}=\varlimsup_{r \rightarrow \infty} \frac{\log \log S_{\theta-\varepsilon, \theta+\varepsilon}(r, E)}{\log r}=+\infty$ for all $\varepsilon>0$.

Proof. Suppose that $f(z)$ is a non-trivial solution of (2.2). Then

$$
\frac{f^{(n)}}{f}+A_{n-2}(z) \frac{f^{(n-2)}}{f}+\cdots+A_{1}(z) \frac{f^{\prime}}{f}+A_{0}(z)=0 .
$$

We apply Wiman-Valiron theory to (2.3). Hence there exists a set $D_{1} \subset$ $[0,+\infty)$ of finite logarithmic measure such that if $r \notin D_{1}$ and $z$ is a point on $|z|=r$ at which $|f(z)|=M(r, f)$, then

$$
\left|\frac{f^{(j)}}{f}\right|=\left(\frac{\nu(r)}{z}\right)^{j}(1+o(1)), \quad j=1,2, \ldots, n,
$$

where $\nu(r)$ denotes the central index of $f(z)$.

It follows from (2.3) and (2.4) that

$$
\begin{aligned}
\nu(r)^{n}(1+ & o(1))+\nu(r)^{n-2} z^{2} A_{n-2}(z)(1+o(1))+\cdots \\
& +\nu(r) z^{n-1} A_{1}(z)(1+o(1))+z^{n} A_{0}(z)=0 .
\end{aligned}
$$

Set $\sigma_{2}=\max _{0 \leq j \leq n-2}\left\{\sigma_{2}\left(A_{j}\right)\right\}$. For all arbitrary $\varepsilon>0$, there exists a set $D_{2} \subset(1,+\infty)$ of finite logarithmic measure such that

$$
\left|A_{j}(z)\right| \leq \exp \left\{\exp \left(r^{\sigma_{2}+\varepsilon}\right)\right\}, \quad j=0,1,2, \ldots, n-2,
$$

when $|z| \notin[0,1] \cup D_{2}$ and $r \rightarrow+\infty$. 
It follows from (2.5) and (2.6) that

$$
\nu(r) \leq n r^{n} \exp \left\{\exp \left(r^{\sigma_{2}+\varepsilon}\right)\right\} \leq \exp \left\{\exp \left(r^{\sigma_{2}+2 \varepsilon}\right)\right\} .
$$

Since $f(z)$ is analytic, $f(z)$ satisfies the condition of Lemma 2.1. Thus, (2.1) and (2.7) implies that

$$
\varlimsup_{r \rightarrow+\infty} \frac{\log \log \log T(r, f)}{\log r} \leq \sigma_{2} .
$$

Now we suppose that $f_{1}, f_{2}, \ldots, f_{n}$ be $n$ linearly independent solutions of (2.2). Set $E=f_{1} f_{2} \cdots f_{n}$, and Wronskian determinant

$$
W=W\left(f_{1}, f_{2}, \ldots, f_{n}\right)=\left|\begin{array}{rrrr}
f_{1} & f_{2} & \cdots & f_{n} \\
f_{1}^{\prime} & f_{2}^{\prime} & \cdots & f_{n}^{\prime} \\
\vdots & \vdots & \ddots & \vdots \\
f_{1}^{(n-1)} & f_{2}^{(n-1)} & \cdots & f_{n}^{(n-1)}
\end{array}\right| .
$$

It follows from Lemma 2.2, without loss of generality, we can set

$$
W\left(f_{1}, f_{2}, \ldots, f_{n}\right)=1 \text {. }
$$

From (2.8), we have

$$
\varlimsup_{r \rightarrow+\infty} \frac{\log \log \log T\left(r, f_{j}\right)}{\log r} \leq \sigma_{2}, \quad j=1,2, \ldots, n .
$$

Hence

$$
\varlimsup_{r \rightarrow+\infty} \frac{\log \log \log T(r, E)}{\log r} \leq \sigma_{2} .
$$

Now dividing (2.9) by $E$, we have

$$
\begin{aligned}
\frac{1}{E}=\frac{W}{E} & =\left|\begin{array}{rrrr}
1 & 1 & \cdots & 1 \\
\frac{f_{1}^{\prime}}{f_{1}} & \frac{f_{2}^{\prime}}{f_{2}} & \cdots & \frac{f_{n}^{\prime}}{f_{n}} \\
\vdots & \vdots & \ddots & \vdots \\
\frac{f_{1}^{(n-1)}}{f_{1}} & \frac{f_{2}^{(n-1)}}{f_{2}} & \cdots & \frac{f_{n}^{(n-1)}}{f_{n}}
\end{array}\right| \\
& =\sum_{1 \leq j_{s} \neq j_{1} \leq n}(-1)^{\tau\left(j_{1}, j_{2}, \ldots, j_{n}\right)} \cdot 1_{j_{1}} \cdot \frac{f_{j_{2}}^{\prime}}{f_{j_{2}}} \cdot \frac{f_{j_{3}}^{\prime \prime}}{f_{j_{3}}} \cdots \frac{f_{j_{s}}^{(s-1)}}{f_{j_{s}}} \cdots \frac{f_{j_{n}}^{(n-1)}}{f_{j_{n}}} \\
& =\sum_{1 \leq j_{s} \neq j_{1} \leq n}(-1)^{\tau\left(j_{1}, j_{2}, \ldots, j_{n}\right)} \prod_{s=2}^{n} \frac{f_{j_{s}}^{(s-1)}}{f_{j_{s}}},
\end{aligned}
$$

where $1_{j_{1}}$ denotes the number 1 in row 1 and in column $j_{1}$ and $\tau\left(j_{1}, j_{2}, \ldots, j_{n}\right)$ denotes the inverse order number of $j_{1}, j_{2}, \ldots, j_{n}$, and $j_{1}, j_{2}, \ldots, j_{n}$ is an arrangement of $1,2, \ldots, n$. 
We deduce from (2.10) and Lemma 2.3 (ii) in which we set $R=2 r$ that, for $j=1,2, \ldots, n$ and for all sufficiently small $\varepsilon>0$,

$$
\begin{aligned}
A_{\theta-\varepsilon, \theta+\varepsilon}\left(r, \frac{f_{j}^{\prime}}{f_{j}}\right) & \leq K \int_{1}^{2 r} \frac{\log T\left(r, f_{j}\right)}{t^{1+k}} d t+O(1) \\
& \leq K \int_{1}^{2 r} \frac{\exp \left(t^{\sigma_{2}+\varepsilon}\right)}{t^{1+\frac{\pi}{2 \varepsilon}}} d t+O(1) \leq K \exp \left((2 r)^{\sigma_{2}+\varepsilon}\right),
\end{aligned}
$$

where $K$ is a sufficiently large positive constant and the following $K$ is the same but can be different.

Since, for $j=1,2, \ldots, n$ and for all sufficiently small $\varepsilon>0$,

$$
m\left(r, \frac{f_{j}^{\prime}}{f_{j}}\right)=O\left(\log T\left(2 r, f_{j}\right)+\log r\right) \leq K \exp \left((2 r)^{\sigma_{2}+\varepsilon}\right),
$$

we deduce from Lemma 2.3 (ii) that, for $j=1,2, \ldots, n$ and for all sufficiently small $\varepsilon>0$,

$$
B_{\theta-\varepsilon, \theta+\varepsilon}\left(r, \frac{f_{j}^{\prime}}{f_{j}}\right) \leq K \exp \left((2 r)^{\sigma_{2}+\varepsilon}\right) .
$$

Therefore we have, for all sufficiently small $\varepsilon>0$,

$$
D_{\theta-\varepsilon, \theta+\varepsilon}\left(r, \frac{f_{j}^{\prime}}{f_{j}}\right) \leq K \exp \left((2 r)^{\sigma_{2}+\varepsilon}\right), \quad j=1,2, \ldots, n .
$$

Similarly, we have, for $j=1,2, \ldots, n$ and for all sufficiently small $\varepsilon>0$,

$$
D_{\theta-\varepsilon, \theta+\varepsilon}\left(r, \frac{f_{j}^{(s)}}{f_{j}}\right) \leq \sum_{l=1}^{s} D_{\theta-\varepsilon, \theta+\varepsilon}\left(r, \frac{f_{j}^{(l)}}{f_{j}^{(l-1)}}\right) \leq K \exp \left((2 r)^{\sigma_{2}+\varepsilon}\right) .
$$

It follows from (2.12) and (2.14) that

$$
\begin{aligned}
D_{\theta-\varepsilon, \theta+\varepsilon}\left(r, \frac{1}{E}\right) & =D_{\theta-\varepsilon, \theta+\varepsilon}\left(r, \sum_{1 \leq j_{s} \neq j_{1} \leq n}(-1)^{\tau\left(j_{1}, j_{2}, \ldots, j_{n}\right)} \prod_{s=2}^{n} \frac{f_{j_{s}}^{(s-1)}}{f_{j_{s}}}\right) \\
& \leq K \exp \left((2 r)^{\sigma_{2}+\varepsilon}\right)
\end{aligned}
$$

for all sufficiently small $\varepsilon>0$.

Since, by Lemma 2.3 (i),

$$
\begin{aligned}
S_{\theta-\varepsilon, \theta+\varepsilon}(r, E) & =S_{\theta-\varepsilon, \theta+\varepsilon}\left(r, \frac{1}{E}\right)+O(1) \\
& =D_{\theta-\varepsilon, \theta+\varepsilon}\left(r, \frac{1}{E}\right)+C_{\theta-\varepsilon, \theta+\varepsilon}\left(r, \frac{1}{E}\right)+O(1),
\end{aligned}
$$

we have, for all sufficiently small $\varepsilon>0$,

$$
S_{\theta-\varepsilon, \theta+\varepsilon}(r, E) \leq K\left\{C_{\theta-\varepsilon, \theta+\varepsilon}\left(r, \frac{1}{E}\right)+\exp \left((2 r)^{\sigma_{2}+\varepsilon}\right)\right\} .
$$


Sufficiency. Now we suppose that $\theta_{0} \in \mathbb{R}$ such that for any sufficiently small $\varepsilon>0$,

$$
\begin{aligned}
& \varlimsup_{r \rightarrow \infty} \frac{\log \log \log M\left(\Omega\left(\left(\theta_{0}-\frac{\varepsilon}{3}, \theta_{0}+\frac{\varepsilon}{3}\right), r\right), E\right)}{\log r} \\
= & \varlimsup_{r \rightarrow \infty} \frac{\log \log S_{\theta_{0}-\frac{\varepsilon}{3}, \theta_{0}+\frac{\varepsilon}{3}}(r, E)}{\log r}=+\infty .
\end{aligned}
$$

Together with (2.11) and $\sigma_{2}\left(A_{j}\right)=0(j=0,1,2, \ldots, n-2), E$ satisfies the conditions of Lemma 2.4. Thus, we can find a finite complex $a$ such that

$$
\varlimsup_{r \rightarrow \infty} \frac{\log \log n\left(\Omega\left(\left(\theta_{0}-\frac{2 \varepsilon}{3}, \theta_{0}+\frac{2 \varepsilon}{3}\right), r\right), E=a\right)}{\log r}=+\infty .
$$

For any given $M>\frac{\pi}{2 \varepsilon}$, we deduce from (2.16) that there exists a sequence $\left\{r_{n}\right\}$ of real numbers with $r_{n} \rightarrow+\infty(n \rightarrow+\infty)$ such that for every $\varepsilon>0$ we have

$$
n\left(\Omega\left(\left(\theta_{0}-\frac{2 \varepsilon}{3}, \theta_{0}+\frac{2 \varepsilon}{3}\right), r_{n}\right), E=a\right) \geq \exp \left(r_{n}^{M}\right) .
$$

Suppose that $a_{v}=\left|a_{v}\right| e^{i \alpha_{v}}(v=1,2, \ldots)$ are the roots of $E=a$, counting multiplicities, in the angular domain $\Omega\left(\theta_{0}-\varepsilon, \theta_{0}+\varepsilon\right)$. To compute $\sigma_{2}\left(\Omega\left(\theta_{0}-\right.\right.$ $\left.\left.\varepsilon, \theta_{0}+\varepsilon\right), E\right)$, we first observe that $\theta_{0}-\frac{2 \varepsilon}{3}<\alpha_{v}<\theta_{0}+\frac{2 \varepsilon}{3}$ implies for $k=\frac{\pi}{2 \varepsilon}$ the inequalities

Hence

$$
k \cdot \frac{\varepsilon}{3}<k\left(\alpha_{v}-\theta_{0}+\varepsilon\right)<\pi-k \cdot \frac{\varepsilon}{3} .
$$

$$
\sin k\left(\alpha_{v}-\theta_{0}+\varepsilon\right) \geq \sin \left(k \cdot \frac{\varepsilon}{3}\right)=\sin \frac{\pi}{6}=\frac{1}{2} .
$$

Moreover, we write a sum below as a Stieltjes-integral,

$$
\begin{aligned}
\sum\left(\frac{1}{\left|a_{v}\right|^{k}}-\frac{\left|a_{v}\right|^{k}}{\left(2 r_{n}\right)^{2 k}}\right) & =\sum\left(\frac{1}{\left|a_{v}\right|^{k}}\right)-\sum\left(\frac{\left|a_{v}\right|^{k}}{\left(2 r_{n}\right)^{2 k}}\right) \\
& =\int_{1}^{r_{n}} \frac{d n(t)}{t^{k}}-\frac{1}{\left(2 r_{n}\right)^{2 k}} \int_{1}^{r_{n}} t^{k} d n(t),
\end{aligned}
$$

where a short hand notation $n(t)=n\left(\Omega\left(\theta_{0}-\frac{2 \varepsilon}{3}, \theta_{0}+\frac{2 \varepsilon}{3}\right), E=a\right)$ will be used.

Application of Lemma 2.3(i), (2.17), (2.18), the partial integration of the above Stieltjes-integral and the definition of $S_{\alpha, \beta}(r, E)$ now results in

$$
\begin{aligned}
& S_{\theta_{0}-\varepsilon, \theta_{0}+\varepsilon}\left(2 r_{n}, E\right) \\
= & S_{\theta_{0}-\varepsilon, \theta_{0}+\varepsilon}\left(2 r_{n}, \frac{1}{E-a}\right)+O(1) \\
\geq & C_{\theta_{0}-\varepsilon, \theta_{0}+\varepsilon}\left(2 r_{n}, \frac{1}{E-a}\right)+O(1) \\
= & 2 \sum_{1<\left|a_{v}\right|<2 r_{n}}\left(\frac{1}{\left|a_{v}\right|^{k}}-\frac{\left|a_{v}\right|^{k}}{\left(2 r_{n}\right)^{2 k}}\right) \sin k\left(\alpha_{v}-\theta_{0}+\varepsilon\right)+O(1)
\end{aligned}
$$




$$
\begin{aligned}
& \geq 2 \sum_{\substack{1<\left|a_{v}\right|<r_{n} \\
\theta_{0}-\frac{2 \varepsilon}{3}<\alpha_{v}<\theta_{0}+\frac{2 \varepsilon}{3}}}\left(\frac{1}{\left|a_{v}\right|^{k}}-\frac{\left|a_{v}\right|^{k}}{\left(2 r_{n}\right)^{2 k}}\right) \sin \left(k \cdot \frac{\varepsilon}{3}\right)+O(1) \\
& =2\left\{\int_{1}^{r_{n}} \frac{d n(t)}{t^{k}}-\frac{1}{\left(2 r_{n}\right)^{2 k}} \int_{1}^{r_{n}} t^{k} d n(t)\right\} \sin \frac{\pi}{6}+O(1) \\
& =\frac{n\left(r_{n}\right)}{r_{n}^{k}}+k \int_{1}^{r_{n}} \frac{n(t)}{t^{1+k}} d t-\frac{r_{n}^{k} n\left(r_{n}\right)}{\left(2 r_{n}\right)^{2 k}}+\frac{k}{\left(2 r_{n}\right)^{2 k}} \int_{1}^{r_{n}} t^{k-1} n(t) d t+O(1) \\
& \geq\left(1-\frac{1}{2^{2 k}}\right) \frac{n\left(r_{n}\right)}{r_{n}^{k}}+O(1) \geq \exp \left(r_{n}^{M-\varepsilon}\right),
\end{aligned}
$$

where $n(t)$ is the numbers of the roots of the equation $E(z)=a$, counting multiplicities, in the sector $\Omega\left(\left(\theta_{0}-\frac{2 \varepsilon}{3}, \theta_{0}+\frac{2 \varepsilon}{3}\right), t\right)$. Therefore we have

$$
\varlimsup_{r \rightarrow \infty} \frac{\log \log S_{\theta_{0}-\varepsilon, \theta_{0}+\varepsilon}(r, E)}{\log r} \geq M-\varepsilon .
$$

Since $\varepsilon$ can be arbitrary small and $M$ be arbitrary large, it follows from (2.15), (2.20) and $\sigma_{2}\left(A_{j}\right)=0(j=0,1,2, \ldots, n-2)$ that $\lambda_{2, \theta_{0}}(E)=+\infty$.

Necessary. Suppose that $\lambda_{2, \theta_{0}}(E)=+\infty$. Similar to the proof of (2.19), we have

$$
S_{\theta_{0}-\varepsilon, \theta_{0}+\varepsilon}(2 r, E) \geq\left(1-\frac{1}{2^{2 k}}\right) \frac{n(r)}{r^{k}}+O(1),
$$

where $n(r)=n\left(\Omega\left(\left(\theta_{0}-\frac{2 \varepsilon}{3}, \theta_{0}+\frac{2 \varepsilon}{3}\right), r\right), E=a\right), k=\frac{\pi}{2 \varepsilon}$.

By the Maximum Modulus Theorem, we have

$$
\begin{aligned}
& S_{\theta_{0}-\varepsilon, \theta_{0}+\varepsilon}(2 r, E) \\
= & A_{\theta_{0}-\varepsilon, \theta_{0}+\varepsilon}(2 r, E)+B_{\theta_{0}-\varepsilon, \theta_{0}+\varepsilon}(2 r, E) \\
= & \frac{k}{\pi} \int_{1}^{r}\left(\frac{1}{t^{k}}-\frac{t^{k}}{r^{2 k}}\right)\left\{\log ^{+}\left|E\left(t e^{i\left(\theta_{0}-\varepsilon\right)}\right)\right|+\log ^{+}\left|E\left(t e^{i\left(\theta_{0}+\varepsilon\right)}\right)\right|\right\} \frac{d t}{t} \\
& +\frac{2 k}{\pi r^{k}} \int_{\theta_{0}-\varepsilon}^{\theta_{0}+\varepsilon} \log ^{+}\left|g\left(r e^{i \theta}\right)\right| \sin k\left(\theta-\theta_{0}+\varepsilon\right) d \theta \\
\leq & \log ^{+} M\left(\Omega\left(\left(\theta_{0}-\varepsilon, \theta_{0}+\varepsilon\right), r\right), E\right)\left\{\frac{2 k}{\pi} \int_{1}^{r} \frac{1}{t^{k+1}} d t+\frac{2 k}{\pi r^{k}} \int_{\theta_{0}-\varepsilon}^{\theta_{0}+\varepsilon} d \theta\right\} \\
\leq & 2 \log ^{+} M\left(\Omega\left(\left(\theta_{0}-\varepsilon, \theta_{0}+\varepsilon\right), r\right), E\right),
\end{aligned}
$$

where $k=\frac{\pi}{2 \varepsilon}, r \geq 1$.

It follows from $(2.21),(2.22)$ and $\lambda_{2, \theta_{0}}=+\infty$ that

$$
\begin{aligned}
\varlimsup_{r \rightarrow \infty} \frac{\log \log \log M\left(\Omega\left(\left(\theta_{0}-\varepsilon, \theta_{0}+\varepsilon\right), r\right), E\right)}{\log r} & =\varlimsup_{r \rightarrow \infty} \frac{\log \log S_{\theta_{0}-\varepsilon, \theta_{0}+\varepsilon}(r, E)}{\log r} \\
& =+\infty .
\end{aligned}
$$

The proof of Theorem 2.5 is completed. 


\section{A relation between a cluster ray of zeros and Borel direction}

We begin with some preparations.

Let $g(z)$ be an entire function with the hyper order $\rho(0<\rho \leq+\infty)$. A ray $L: \arg z=\theta_{0}\left(0 \leq \theta_{0}<2 \pi\right)$ is called a cluster ray of zeros of $g=a$ with hyper order $\lambda_{2, \theta_{0}}(g)$, if for any small positive number $\varepsilon$, we have

$$
\varlimsup_{r \rightarrow+\infty} \frac{\log \log n\left(\Omega\left(\left(\theta_{0}-\varepsilon, \theta_{0}+\varepsilon\right), r\right), g=a\right)}{\log r}=\lambda_{2, \theta_{0}}(g) .
$$

It is similar to the proof of Theorem 3.11 in [13], we have:

Lemma 3.1. Suppose that $g(z)$ is meromorphic in $\mathbb{C}$ with the hyper order $\rho(0<\rho \leq+\infty)$. If

$$
B: \quad \arg z=\theta_{0}, \quad 0 \leq \theta_{0}<2 \pi,
$$

is a Borel direction of $g(z)$ with the hyper order $\rho$, then there exists a sequence of filling disks $\Gamma_{j}$ of $g(z)$ with the hyper order $\rho$ such that

$\Gamma_{j}:\left|z-z_{j}\right|<\varepsilon_{j}\left|z_{j}\right|(j=1,2, \ldots), z_{j}=\left|z_{j}\right| e^{i \theta_{0}}, \lim _{j \rightarrow+\infty}\left|z_{j}\right|=+\infty, \lim _{j \rightarrow+\infty} \varepsilon_{j}=0$, and $g(z)$ takes every complex value in $\Gamma_{j}$ at least $n_{j} \geq \exp \left\{\left|z_{j}\right|^{\rho_{j}}\right\}$ times, except for some values which can be covered in two small disks with radii $\exp \left\{-n_{j}\right\}$ on the Riemann sphere, where $\rho_{j} \rightarrow \rho(j \rightarrow+\infty)$.

The above disks of Lemma 3.1 are called filling disks of $g(z)$ with the hyper order $\rho$.

Lemma $3.2([7])$. Suppose that $\Omega(\alpha, \beta)$ and $\Omega\left(\alpha^{\prime}, \beta^{\prime}\right)$ are two angular domains such that $\alpha<\alpha^{\prime}<\beta^{\prime}<\beta$ and that $g(z)$ is an entire function and satisfies the hypotheses of Lemma 2.5. Then $g(z)$ has a Borel direction with hyper order $\sigma_{2}\left(\Omega\left(\alpha^{\prime}, \beta^{\prime}\right), g\right)$ in the angular domain $\Omega\left(\alpha^{\prime}, \beta^{\prime}\right)$.

As for a relation between a cluster ray of zeros and Borel direction, similar to Theorem 1.2, we have the following result.

Theorem 3.3. Let $A_{j}(z)(j=0,1, \ldots, n-1)$ be entire functions with order $\sigma\left(A_{j}\right)=+\infty$ and the hyper order $\sigma_{2}\left(A_{j}\right)=0(j=0,1,2, \ldots, n-2)$, and let $f_{1}, f_{2}, \ldots, f_{n}$ be $n$ linearly independent solutions of (2.2). Set $E=f_{1} f_{2} \cdots f_{n}$. Suppose that the hyper order exponent of convergence of zeros sequence of $E$ is $+\infty$. Then a ray $\arg z=\theta$ from the origin is a Borel direction of $E$ with the hyper order $+\infty$ and $\rho_{2}(\Omega(\theta-\varepsilon, \theta+\varepsilon), E)=+\infty$ if and only if $\lambda_{2, \theta}(E)=+\infty$.

Proof. It is well know that $\sigma_{2}(E)=+\infty$ in this case.

Necessary. We suppose that ray $\arg z=\theta_{0}$ is a Borel direction of $E$ with the hyper order $\rho=+\infty$. We then prove that a ray $\arg z=\theta_{0}$ is a cluster ray of zeros of $E$ with the hyper order $\rho=+\infty$.

By Lemma 3.1, there exists a sequence of filling $\operatorname{discs} \Gamma_{j}$ of $E$ with the hyper order $\rho=+\infty$ such that

$\Gamma_{j}:\left|z-z_{j}\right|<\varepsilon_{j}\left|z_{j}\right|, z_{j}=\left|z_{j}\right| e^{i \theta_{0}}, \lim _{j \rightarrow+\infty}\left|z_{j}\right|=+\infty, \lim _{j \rightarrow+\infty} \varepsilon_{j}=0, j=1,2, \ldots$ 
and $g(z)$ takes every complex value in $\Gamma_{j}$ at least $n_{j} \geq \exp \left\{\left|z_{j}\right|^{\rho_{j}}\right\}$ times, except for some values which can be covered in two small disks with radii $\exp \left\{-n_{j}\right\}$ on the Riemann sphere, where $\rho_{j} \rightarrow \rho(j \rightarrow+\infty)$.

For any given $\varepsilon>0$, we have

$$
\Gamma_{j} \subset \Omega_{\varepsilon}\left(\theta_{0}-\varepsilon, \theta_{0}+\varepsilon\right)
$$

for all sufficiently large $j$.

Noting $E(z)$ is an entire function, $\infty$ is a Picard exceptional value. Therefore, $\infty$ lies in one of two small spherical disks in the definition of filling disks.

Let us denote the spherical distance of $z_{1}, z_{2}$ by $\left|z_{1}, z_{2}\right|$. Therefore, for all sufficiently large $j$, we can find a point $a_{j} \in \Gamma_{j}$ such that

$$
\left|E\left(a_{j}\right), \infty\right|=\frac{1}{\left(1+\left|E\left(a_{j}\right)\right|^{2}\right)^{\frac{1}{2}}} \leq 2 \exp \left\{-n_{j}\right\} .
$$

Thus we can find a positive constant $c$ not dependent on $j$ such that

$$
\left|E\left(a_{j}\right)\right|>c \exp \left\{n_{j}\right\} \geq c \exp \left\{\exp \left(\left|z_{j}\right|^{\rho_{j}}\right)\right\}
$$

for all sufficiently large $j$.

Noting $\left|a_{j}\right|=(1+o(1))\left|z_{j}\right|$ and $M$ is arbitrary sufficiently large, we have

$$
\varlimsup_{r \rightarrow+\infty} \frac{\log \log \log M\left(\Omega\left(\theta_{0}-\varepsilon, \theta_{0}+\varepsilon\right) \cap \bar{D}(0, r), E\right)}{\log r}=+\infty,
$$

where $\bar{D}(0, r)=\{z ;|z| \leq r\}$.

Together with the hypotheses of Theorem 3.3, $E$ satisfies the sufficient condition of Theorem 2.5. Thus, we have $\lambda_{2, \theta_{0}}(E)=+\infty$, i.e., $\arg z=\theta_{0}$ is a cluster ray of zeros of $E$ with hyper order $+\infty$.

Sufficiency. Suppose that $\arg z=\theta_{0}$ is a ray such that $\lambda_{2, \theta_{0}}(E)=+\infty$. By Theorem 2.5 again, we have

$$
\begin{aligned}
\lim _{r \rightarrow+\infty} \frac{\log \log \log M\left(\Omega\left(\left(\theta_{0}-\varepsilon, \theta_{0}+\varepsilon\right), r\right), E\right)}{\log r} & =\lim _{r \rightarrow+\infty} \frac{\log \log S_{\theta_{0}-\varepsilon, \theta_{0}+\varepsilon}(r, E)}{\log r} \\
& =+\infty
\end{aligned}
$$

for all sufficiently small $\varepsilon>0$.

It follows from $(2.11)$ and $\sigma_{2}\left(A_{j}\right)=0(j=0,1,2, \ldots, n-2)$ that $E$ satisfies the conditions of Lemma 3.2. Hence $E(z)$ has a Borel direction with the hyper order $+\infty$ in the angular domain $\Omega\left(\theta_{0}-\varepsilon, \theta_{0}+\varepsilon\right)$. Since $\varepsilon$ is arbitrary, the ray $\arg z=\theta_{0}$ is a Borel direction of $E$ with the hyper order $+\infty$. This concludes the proof of Theorem 3.3.

Acknowledgement. The authors would like to thank the referees for valuable suggestions to improve our paper. 


\section{References}

[1] Z. X. Chen and C. C. Yang, On the zeros and hyper-order of meromorphic solutions of linear differential equations, Ann. Acad. Sci. Fenn. Math. 24 (1999), no. 1, 215-224.

[2] Z. X. Chen and K. H. Shon, On the growth of solutions of a class of higher order differential equations, Acta Math. Sci. Ser. B Engl. Ed. 24 (2004), no. 1, 52-60.

[3] A. A. Gol'dberg and I. V. Ostrovskii, The Distribution of Values of Meromorphic Functions, Izdat. Nauka, Moscow, 1970.

[4] S. A. Gao, Z. X. Chen, and T. W. Chen, Oscillation theory of linear differential equation, Huazhong University of Science and Technology Press, 1998.

[5] W. K. Hayman, Meromorphic Function, Oxford, 1964.

[6] Y. Z. He and X. Z. Xiao, Algebroid functions and ordinary differential equations, Science Press, Beijing, 1998

[7] Z. B. Huang and Z. X. Chen, Angular distribution with hyper-order in complex oscillation theory, (Chinese) Acta Math. Sinica (Chin. Ser.) 50 (2007), no. 3, 601-614.

[8] I. Laine, Nevanlinna Theory and Complex Differential Equations, Walter de Gruyter \& Co., Berlin, 1993.

[9] R. Nevanlinna, Über die Eigenschaften meromorpher funktionen in einem winkelraum, Acta Soc. Sci. Fenn. 50 (1925), 1-45.

[10] S. J. Wu, On the location of zeros of solutions of $f^{\prime \prime}+A(z) f=0$ where $A(z)$ is entire, Math. Scand. 74 (1994), no. 2, 293-312.

[11] _ Angular distribution in complex oscillation theory, Sci. China Ser. A 48 (2005), no. $1,107-114$

[12] Z. J. Wu and D. C. Sun, Angular distribution in complex oscillation, Acta Math. Sinica (Chin. Ser.) 50 (2007), no. 6, 1297-1304.

[13] L. Yang, Value Distribution Theory and its New Researches, Beijing Science Press, 1992.

[14] C. F. Yi, Angular distribution of solutions of a higher-order differential equation, Acta Math. Sinica (Chin. Ser.) 48 (2005), no. 1, 133-140.

Zhibo Huang

School of Mathematical Sciences

South China Normal University

Guangzhou 510631, P. R. China

E-mail address: hzbo20019@sina.com

ZongXuan Chen

School of Mathematical Sciences

South China Normal University

Guangzhou 510631, P. R. China

E-mail address: chzx@vip.sina.com 